\title{
A VISITA PRÉ-OPERATÓRIA COMO FATOR ATENUANTE DA ANSIEDADE EM PACIENTES CIRÚRGICOS
}

\author{
The preoperative visit as the anxiety mitigating factor in surgical patients \\ La visita preoperatoria como un factor atenuante de la ansiedad en pacientes quirúrgicos \\ Thiago Franco Gonçalves', Veronica Cecilia Calbo de Medeiros ${ }^{2}$
}

RESUMO: Objetivo: Identificar se a realização da visita pré-operatória seria um fator que possibilita minimizar o nível de ansiedade apresentado por pacientes cirúrgicos. Método: Pesquisa de caráter exploratório prospectivo, com amostra de 20 pacientes que foram submetidas ao procedimento de histerectomia total ou parcial, por qualquer técnica cirúrgica. Resultados: Após a aplicação do Inventário de Ansiedade Traço-Estado, foi identificado que o grupo controle apresentou nível de ansiedade superior quando comparado ao grupo pesquisa. Conclusão: A visita de enfermagem pré-operatória contribuiu para que o nível de ansiedade seja inferior nos que a recebem.

Palavras-chave: Histerectomia. Enfermagem perioperatória. Ansiedade. Período pré-operatório.

ABSTRACT: Objective: To identify if the preoperative visit would be a factor that enables us to minimize the level of anxiety in surgical patients. Method: Exploratory prospective research with 20 patients who were subjected to total or partial hysterectomy, for any surgical technique. Results: After the application of the State-Trait Anxiety Inventory, it was identified that the control group presented higher anxiety levels when compared to the research group. Conclusion: Preoperative nursing visits contributed to the lower level of anxiety among those who receive it.

Keywords: Hysterectomy. Perioperative nursing. Anxiety. Preoperative period.

RESUMEN: Objetivo: Identificar si la realización de la visita preoperatoria sería un factor que posibilitaría la minimización del nivel de ansiedad presentado por los pacientes quirúrgicos. Método: Investigación exploratoria y prospectiva, con una muestra compuesta por 20 pacientes que fueron sometidas al procedimiento de la histerectomía total o parcial por cualquier técnica quirúrgica. Resultados: Después de la aplicación del Inventario de la Ansiedad Seguimiento-Estado, se identificó que el Grupo Control presentó un mayor nivel de ansiedad comparado al grupo de la investigación. Conclusión: La visita preoperatoria de enfermería contribuye para que el nivel de ansiedad sea menor en los pacientes que la reciben.

Palabras clave: Histerectomía. Enfermería perioperatoria. Ansiedad. Período preoperatorio.

'Enfermeiro. Especialista em Enfermagem em Centro Cirúrgico. Centro Cirúrgico do Hospital e Maternidade São Luiz, Unidade Brasil. E-mail: thigo.enf@hotmail.com Rua Savigni, 199 - Vila Alpina - CEP: $03203-030$ - São Paulo (SP), Brasil.

Enfermeira. Mestre em Saúde do Adulto. Docente do Centro Universitário São Camilo. E-mail: veronicacalbo@terra.com.br

Recebido: 13 nov. 2015. Aprovado: 28 mar. 2016

DOI: 10.5327/Z1414-4425201600010004 


\section{INTRODUÇÃO}

Por muitas vezes, os pacientes que são admitidos em instituições de atendimento à saúde para a realização de um procedimento cirúrgico não possuem informações e orientações claras. Essa falta de conhecimento a respeito dos procedimentos, aos quais o paciente irá se submeter, propicia uma série de emoções nos indivíduos ${ }^{1}$.

Sendo assim, essas emoções correlacionam-se diretamente ao sentimento de ansiedade, uma vez que sua definição pela Associação Norte-Americana de Diagnósticos de Enfermagem é:

[...] vago e incômodo sentimento de desconforto ou temor, acompanhado por resposta autonômica (a fonte é frequentemente não específica ou desconhecida para o indivíduo); sentimento de apreensão causado pela antecipação de perigo. E um sinal de alerta que chama a atenção para um perigo iminente e permite ao indivíduo tomar medidas para lidar com a ameaça ${ }^{2}$.

Ansiedade também pode ser definida como "uma sensação de mal-estar psíquico caracterizado pelo temor de um perigo eminente real ou imaginário"3. Já, estresse é qualquer evento, podendo ser real ou imaginário, que afete a estabilidade do organismo mesmo de maneira sutil ${ }^{4}$. Portanto, ambos os conceitos (ansiedade e estresse) podem ser interpretados como sendo correlatos.

A ansiedade, apresentada pelos indivíduos frente a fatores estressantes, pode ser benéfica quando associada ao mecanismo de "luta ou fuga", uma vez que a ativação do sistema nervoso central libera adrenalina e hormônios corticoides para rápida ativação celular; entretanto, em situações como o ato anestésico, essa sensação, devido ao mesmo mecanismo, pode acarretar alterações nos parâmetros dos sinais vitais, como a elevação da pressão arterial, boca seca, sudorese, palpitações, calafrios, vômitos, aumento da frequência respiratória e cardíaca ${ }^{5,6}$.

O fator estressante pode ser interno ou externo, em que haja uma avaliação cognitiva do indivíduo que os classifique como ameaça (estímulo negativo) ou desafio (estímulo positivo) onde a estimulação do organismo ocorre devido à liberação de catecolaminas e de corticosteroides?.

A ativação do hipotálamo, em uma situação de estresse, desencadeia dois tipos de estímulos: um elétrico e outro químico. O estímulo elétrico atua sob a medula adrenal, que é responsável pela liberação de epinefrina na corrente sanguínea; o químico, gerado pelo hormônio adenocorticotrófico (ACTH), ativa a zona fasciculada do córtex adrenal, ocasionando a liberação do cortisol, e prepara o organismo para lutar ou fugir ${ }^{4}$.

Foi descrita a resposta geral de adaptação ao estresse com a sequência de como o estresse se manifesta e interage com o organismo e o ambiente em três fases: o alarme, a resistência e a exaustão8.

Na primeira fase de alerta, o indivíduo sofre sensações que, em alguns casos, não as atribui ao estresse, como palidez, taquicardia e taquipneia, sendo uma tentativa do organismo em suprir os órgãos vitais de aporte sanguíneo e, consequentemente, de energia. Durante a segunda fase de resistência, há a tentativa do organismo em restabelecer a homeostase, na qual, se esse equilíbrio for alcançado, alguns dos sinais e sintomas iniciais podem desaparecer; no entanto, sendo falho, o indivíduo entra na terceira fase, a exaustão ${ }^{8}$.

A exaustão é a incapacidade que o organismo tem de retomar o equilíbrio basal, podendo acarretar danos fisiológicos irreversíveis ${ }^{8}$.

Buscou-se, então, uma forma de quantificar o grau/nível de ansiedade que os indivíduos apresentam.

O Inventário de Ansiedade Traço-Estado (IDATE) resultou de avaliações e seleções dos itens de outras três escalas destinadas à mensuração da ansiedade: a escala manifesta de Taylor, a escala de ansiedade de Welsh e a escala de ansiedade IPAT $(\text { impact }=\text { population } x \text { affluence } x \text { technology })^{9}$.

Nesse inventário, a ansiedade é dividida em dois tipos: o traço e o estado. O traço IDATE-T relaciona-se à propensão do indivíduo em enfrentar a ansiedade durante o cotidiano e o estado IDATE-E é um fato isolado, transitório, em consequência de um momento específico vivenciado ${ }^{10}$.

Em pesquisa realizada ${ }^{11}$ foram avaliados 360 pacientes cirúrgicos. Desses, apenas um paciente não se mostrou ansioso, ao passo que os demais compuseram os grupos de médio (114 indivíduos; $31,7 \%$ ) e alto nível (245 indivíduos; 68,1\%) de ansiedade, com os quais foi utilizada a escala IDATE-E.

O período pré-operatório é o momento ideal para que haja o contato entre enfermeiro e paciente, quando o profissional pode, por meio da visita de enfermagem, passar as informações necessárias sobre o procedimento anestésico-cirúrgico e promover um preparo emocional eficiente e eficaz do paciente, sendo as informações passadas de vital importância para minimizar o nível de ansiedade do mesmo ${ }^{12}$. 
A visita de enfermagem pré-operatória faz parte da sistematização da assistência de enfermagem perioperatória (SAEP), constituindo a primeira fase desse sistema. Entre as atividades realizadas pelo enfermeiro destacam-se o esclarecimento e orientações sobre a cirurgia e a minimização da ansiedade 9 .

Durante a visita de enfermagem, o enfermeiro deve transmitir as informações ao paciente de modo individualizado e com foco nas necessidades que ele apresenta, garantindo que seja passado o que o paciente realmente deseja e precisa saber, mantendo uma sequencia lógica das orientações de maneira que facilite a capacidade de compreensão ${ }^{13}$.

Ao passo que, se houver uma orientação fornecida ao paciente e suas dúvidas forem esclarecidas, as complicações no período pós-operatório poderão ser prevenidas ${ }^{12}$.

A motivação para realização desta pesquisa partiu da vivência prática do autor, com a ansiedade verbalizada pelas pacientes durante a admissão no centro cirúrgico, que gerou a pergunta de pesquisa: Poderia a visita de enfermagem pré-operatória ser um fator que diminuiria a ansiedade dos pacientes?

Com isso, espera-se identificar, com esta pesquisa, que os pacientes que irão receber a visita de enfermagem pré-operatória terão menor nível de ansiedade indicado pela escala IDATE-E.

\section{OBJETIVO}

Identificar se a realização da visita pré-operatória pode ser um fator que possibilite minimizar o nível de ansiedade apresentado por pacientes cirúrgicos.

\section{MÉTODO}

Esta pesquisa trata-se de um trabalho de caráter exploratório prospectivo descritivo.

O local de estudo escolhido foi um hospital particular de grande porte do estado de São Paulo, na região do ABC. O estudo foi realizado após autorização da Diretoria da instituição e aprovação do Comitê de Ética em Pesquisa sob o número do CAAE: 44715115.2.0000.0062, no mês de julho de 2015 .

Com base no exposto, foi escolhido o inventário de ansiedade estado IDATE-E (Anexo 1), já que se adapta melhor à situação do paciente (o momento cirúrgico) e ao objetivo deste estudo. Essa escala é composta por um questionário contendo
20 perguntas simples com respostas pré-estabelecidas: "muito", "bastante", "pouco" e "não"; as pontuações são, respectivamente, 4, 3, 2, 1. Portanto, os valores obtidos variam de 20 a 80 pontos. As pontuações obtidas na realização da escala caracterizam-se em baixa ansiedade ( 20 - 40 pontos), média ansiedade ( $41-60$ pontos) e alta ansiedade ( $61-80$ pontos).

Nos itens de números $1,2,5,8,10,11,15,16,19,20$ da escala de ansiedade, as pontuações devem ser contrárias aos demais. Ou seja, em vez da pontuação seguir o padrão de 1 a 4, deverão ser pontuadas de 4 a 1 (de forma decrescente), pois esses itens do inventário apresentam afirmações opostas aos demais.

Foram estabelecidos critérios de inclusão para a composição da amostra desta pesquisa, sendo eles: procedimento cirúrgico de caráter eletivo; cliente/ paciente internada na instituição; cirurgias de histerectomia total ou parcial; possibilidade de comunicação entre o pesquisador e cliente/ paciente. Para o critério de exclusão, ficaram estabelecidas as pacientes analfabetas.

A escolha de realizar a pesquisa em pacientes que se submeteram ao procedimento de histerectomia partiu da constatação de que essa é uma cirurgia entendida pelas pacientes como mutiladora e que interfere diretamente na feminilidade e sexualidade delas ${ }^{14}$.

A amostra estudada foi composta por 20 clientes / pacientes e que foram distribuídas em dois grupos: um de pesquisa e outro grupo controle, por meio de sorteio prévio, 10 para o grupo de estudo e 10 para o grupo controle. O sorteio procedeu da seguinte maneira: antes do início da coleta de dados foi feita uma tabela com numeração de 1 a 20 (correspondendo ao número de participantes do estudo) e pedaços de papel com as expressões "com visita" e "sem visita", sendo 10 para cada, dobrados de maneira igual e colocados todos em um saco escuro, chacoalhados e em seguida retirados um por vez, definindo assim, a realização ou não da visita. Conforme aceitação da paciente em participar do estudo ela era inserida na listagem, seguindo a ordem numérica. O grupo pesquisa foi formado por pacientes que receberam a visita pré-operatória de enfermagem. Em contrapartida, o grupo controle não a recebeu. A visita ocorreu com um período de uma hora de antecedência do procedimento cirúrgico em local reservado, respeitando e assegurando a privacidade das pacientes. Foi definido esse número de amostra devido ao tempo para a realização da pesquisa.

Em ambos os grupos, as pacientes foram orientadas acerca do objetivo da pesquisa, assegurando o anonimato das envolvidas e sobre a possibilidade de interromperem a 
participação em qualquer momento, sem ônus, de acordo com a Resolução no 466/12 do Conselho Nacional de Saúde do Ministério da Saúde. Foi solicitada a assinatura do Termo de Consentimento Livre e Esclarecido, também assinado pelo pesquisador responsável pelo estudo.

Durante a visita, o enfermeiro pesquisador informou a cliente / paciente a respeito do período que compreende o espaço perioperatório (desde o preparo para o procedimento até o recebimento da alta da sala de recuperação anestésica). A entrevistada foi orientada de forma que poderia interromper a visita a qualquer momento para realizar questionamentos, a fim de serem sanadas todas as suas dúvidas.

A pesquisa foi realizada no período pré-operatório, uma hora antes do procedimento cirúrgico, em duas fases. A primeira fase foi constituída de um questionário social da participante para a identificação de dados pessoais, cujo preenchimento do impresso ficou a cargo do pesquisador, no qual foram perguntados dados (como idade, experiências com outros procedimentos cirúrgicos, escolaridade e intercorrências anestésicas-cirúrgicas) e analisados em comparação com a escala IDATE-E.

A segunda fase da pesquisa foi a aplicação da escala IDATE-E, imediatamente após o término do questionário social, ainda no período pré-operatório. Essa etapa pode ser considerada como a terceira para o grupo de estudo, pois antes da aplicação da escala foi realizada a visita da enfermagem, cujo preenchimento ficou a cargo da participante da pesquisa, visto que foram necessárias respostas de como esta se sente, não podendo, assim, sofrer interferências de terceiros. Caso a participante apresentasse alguma dúvida sobre o teor das afirmações presentes na escala, poderia ser orientada pelo pesquisador; no entanto, este não pode interferir nas respostas. Todas as fases desta pesquisa foram, então, realizadas antes da paciente realizar o procedimento cirúrgico.

\section{RESULTADOS E DISCUSSÃO}

Os resultados sociais coletados com a pesquisa são apresentados separadamente entre os grupos controle e pesquisa para melhor caracterização de cada um. O mesmo se aplica quanto à necessidade da identificação da idade das participantes, uma vez que cada geração sofre influência por diferentes meios de cultura, crenças e formas de acesso à informação.
Sendo assim, as pacientes que compuseram o grupo controle (grupo de pacientes que não receberam a visita de enfermagem) apresentaram idade média de 45,4 anos. No que se refere à escolaridade, o percentual das pacientes com ensino médio foi de $70 \%$, enquanto as com ensino superior incompleto foi $10 \%$, e $20 \%$ com ensino superior completo.

As que realizaram algum procedimento cirúrgico antes da histerectomia somaram $100 \%$ da amostra desse grupo, sendo que apenas $20 \%$ ( 2 pacientes) apresentaram algum tipo de intercorrência anestésico-cirúrgica, sendo elas náusea, vômito e algia.

No grupo pesquisa, a média de idade foi de 43,2 anos; sendo assim, ambos os grupos apresentaram idade média próxima. O grau de escolaridade nesse grupo foi de $40 \% \mathrm{com}$ ensino médio, $20 \%$ com ensino superior incompleto, $30 \%$ com ensino superior completo e $10 \%$ com pós-graduação.

A porcentagem de pacientes que realizaram algum procedimento cirúrgico anterior à histerectomia foi de $100 \%$. Dessas, $10 \%$ apresentaram intercorrências anestésica-cirúrgicas (dispneia) e $90 \%$ negaram tais eventos.

As pacientes do grupo controle apresentaram média de 45,8 pontos no Inventário de Ansiedade Traço-Estado. Sendo assim, esse grupo demonstrou médio nível de ansiedade, enquanto que o grupo pesquisa obteve 36,3 pontos, indicando baixo nível de ansiedade.

O fato de todas as pacientes terem se submetido a algum outro tipo de cirurgia poderia contribuir para que a ansiedade em ambos os grupos fosse mais baixa, uma vez que um dos fatores para um nível de ansiedade elevado é a ameaça do desconhecido, quando considerado o ato anestésico e o ambiente do centro cirúrgico, porém isso não foi observado. Vale dizer que está sendo levado em consideração apenas o fato de não ser a primeira experiência vivida por elas na realização de um procedimento anestésico e por já terem entrado / estado pelo menos uma vez em um centro cirúrgico, mas a especificidade da cirurgia e os motivos pelos quais foi indicada também merecem ser considerados.

A ansiedade é o diagnóstico de enfermagem que se apresenta com maior frequência no período pré-operatório nos pacientes que serão submetidos a procedimentos cirúrgicos, como ficou evidente em estudo realizado ${ }^{15}$, no qual $86,6 \%$ dos pacientes incluídos no trabalho apresentaram esse diagnóstico de enfermagem.

Assim como defendido em outro estudo ${ }^{16}$, constatou-se que as pacientes que recebem informações acerca dos procedimentos aos quais seriam submetidas durante o ato 
anestésico-cirúrgico conseguem diminuir o fator estressante e, consequentemente, o nível de ansiedade.

Essa ideia é corroborada por outro trabalho ${ }^{17}$, que defende a visita de enfermagem pré-operatória como um ato de relevância ao cuidado biopsicossocioespiritual, tornando o procedimento cirúrgico mais tranquilo para o paciente e diminuindo ou prevenindo os fatores estressantes.

Outro estudo ${ }^{18}$ demonstra que a ansiedade apresentada no período pré-operatório atinge $44,3 \%$ dos pacientes.

Sendo assim, é correto afirmar que a visita de enfermagem pré-operatória é um importante instrumento a ser utilizado pelo enfermeiro nesse período, que é respaldado pela Lei do Exercício Profissional de Enfermagem n ${ }^{\circ} 7.498 / 86$, art. 11, inciso I, alínea $\mathrm{i}^{19}$.

Em outro estudo ${ }^{20}$, há menção de que tais orientações devem ser fornecidas no período pré-operatório mediato para melhor assimilação das informações. Entretanto, durante a pesquisa, essa variável não foi percebida, uma vez que a visita de enfermagem foi realizada no período pré-operatório imediato.

Os questionamentos realizados por grande parcela das pacientes do grupo pesquisa durante a visita de enfermagem não estavam relacionados diretamente com o procedimento cirúrgico ou com o ato anestésico, mas, sim, se as mesmas iriam apresentar ganho de peso após a cirurgia, o que evidencia uma preocupação voltada à aparência física e não às possíveis restrições pós-operatórias imediatas e mediatas que a histerectomia pode ocasionar, podendo ser esse fato considerado como um fator limitante da pesquisa, visto que não é abordado no questionário.

\section{CONCLUSÃO}

Com o presente estudo, foi possível identificar que a ansiedade, como diagnóstico de enfermagem, está presente nos pacientes que irão se submeter a procedimentos cirúrgicos e comprovar a hipótese da pesquisa, de que os pacientes que recebem visita de enfermagem pré-operatória apresentam nível de ansiedade inferior aos que não passam por essa fase do processo.

Importante ressaltar que a visita de enfermagem pré-operatória é uma atividade que está inserida no período perioperatório e a não realização da mesma fragiliza o processo e interfere diretamente no paciente, visto que a ansiedade, mesmo sendo de caráter psicológico, atua sobre o organismo, produzindo alterações nos sinais vitais dos indivíduos, podendo ser causa do cancelamento ou suspensão do ato cirúrgico que, por sua vez, pode gerar maior ansiedade e se tornar um ciclo vicioso.

Espera-se, com este trabalho, contribuir para a prática da enfermagem no período pré-operatório quanto ao cuidado emocional do paciente cirúrgico, sendo esse de importância tão relevante quanto o preparo físico.

\section{REFERÊNCIAS}

1. Medeiros VCC, Peniche ACG. A influência da ansiedade nas estratégias de enfrentamento utilizadas no período pré-operatório. Rev Esc Enferm USP. 2006;40(1):86-92.

2. North American Nursing Diagnosis Association. Diagnósticos de enfermagem da NANDA: definições e classificação. Porto Alegre: Artmed; 2010. p. 256.

3. Silva CRL, Silva RCL, Viana DL. Compacto Dicionário llustrado de Saúde. $4^{\mathrm{a}}$ ed. São Caetano do Sul: Yendis; 2009. p. 129.

4. Mourão Júnior CA, Abramov DM. Fisiologia Essencial. Rio de Janeiro: Guanabara Koogan; 2011.
5. Nosow V, Peniche ACG. Paciente cirúrgico ambulatorial: calatonia e ansiedade. Acta Paul Enferm. 2007;20(2):167-7.

6. Sampaio CEP, Ribeiro DA, Marta CB, Seabra Junior HC, Rose E, Francisco MTR. Fatores determinantes da ansiedade e mecanismos de coping em procedimentos cirúrgicos gerais. Rev Pesq Cuid Fundam (Online). 2013;5(4):547-55.

7. Bianchi ERF. Escala Bianchi de stress. Rev Esc Enferm USP. 2009;43:1055-62.

8. Almeida NDV. Considerações acerca da incidência do estresse em motoristas profissionais. Rev Psicol. 2010;1(1):75-84. 
9. Frias TFP, Costa CMA, Sampaio CEP. 0 impacto da visita pré-operatória de enfermagem no nível de ansiedade de pacientes cirúrgicos. REME Rev Min Enferm. 2010;14(3):345-52.

10. Fioravanti ACM, Santos LF, Maissonette S, Cruz APM, Fernandez JL. Avaliação da estrutura fatorial da Escala de Ansiedade-Traço do IDATE. Aval Psicol. 2006;5(2):217-24.

11. Agostini P, Sakae TM, Feldens VP. Prevalência de sintomas ansiosos em pacientes em consultório de anestesia de Tubarão. Arq Catarin Med. 2011; 40(1):52-6.

12. Silva WV, Nakata S. Comunicação: uma necessidade percebida no período pré-operatório de pacientes cirúrgicos. Rev Bras Enferm. 2005;58(6):673-6.

13. Kruse MHL, Almeida MA, Keretzky KB, Rodrigues E, Silva FP, Schenini FS, et al. Orientação pré-operatória da enfermeira: lembranças de pacientes. Rev Eletr Enf. 2009;11(3):494-500.

14. Schaffir J, Fleming M, Waddell V. Patient perceptions regarding effect of gynecological surgery on sexuality. J Sex Med. 2010;7(2 Pt 1):826-31.
15. Flório MCS, Galvão CM. Cirurgia ambulatorial: identificação dos diagnósticos de enfermagem no período perioperatório. Rev Latino-Am Enf. 2003;11(5):630-7.

16. Santos J, Henckmeier L, BenedetSA. O impacto da orientação pré-operatória na recuperação do paciente cirúrgico. Enferm Foco. 2011;2(3):184-7.

17. Souza LR, Souza MAG, Pinto AS, Cortez EA, Carmos TG, Nascimento RM. Os benefícios da visita pré-operatória de enfermagem para o cliente ciúrgico: revisão sistemática de literatura. Rev Pesqui Cuid Fundam (Online). 2010;2(2):797-806.

18. Marcolino JAM, Suzuki FM, Alli LAC, Gozzani JL, Mathias LAST. Medida da ansiedade e da depressão em pacientes no pré-operatório: estudo comparativo. Rev Bras Anestesiol. 2007;57(2):157-66.

19. Brasil. Lei no 7.498, de 25 de junho de 1986. Dispõe sobre a regulamentação do exercício da enfermagem, e dá outras providências. Brasília: Diário Oficial da União, 26 jun. 1986. Seção I, p. 9.273-9.275.

20. Perrando M, Beuter M, Brondani CM, Roso CC, Santos TM, Predebon GR. $O$ preparo pré-operatório na ótica do paciente cirúrgico. REUFSM. 2011;1(1):61-70.

Anexo 1. Questionário de ansiedade.

Leia as questões a seguir e responda, marcando um $\mathrm{X}$ na coluna que indica melhor como você se sente agora, antes da realização do procedimento cirúrgico.

\begin{tabular}{|c|c|c|c|c|c|}
\hline No & Questão & Não & Pouco & Bastante & Muito \\
\hline 01 & Sinto-me calma & & & & \\
\hline 02 & Sinto-me segura & & & & \\
\hline 03 & Estou calma & & & & \\
\hline 04 & Estou arrependida & & & & \\
\hline 05 & Sinto-me à vontade & & & & \\
\hline 06 & Sinto-me perturbada & & & & \\
\hline 07 & Estou preocupada com possíveis infortúnios & & & & \\
\hline 08 & Sinto-me descansada & & & & \\
\hline 09 & Sinto-me ansiosa & & & & \\
\hline 10 & Sinto-me "em casa" & & & & \\
\hline 11 & Sinto-me confiante & & & & \\
\hline 12 & Sinto-me nervosa & & & & \\
\hline 13 & Estou agitada & & & & \\
\hline 14 & Sinto-me uma "pilha de nervos" & & & & \\
\hline 15 & Estou descontraída & & & & \\
\hline 16 & Sinto-me satisfeita & & & & \\
\hline 17 & Estou preocupada & & & & \\
\hline 18 & Sinto-me superexcitada e confusa & & & & \\
\hline 19 & Sinto-me alegre & & & & \\
\hline 20 & Sinto-me bem & & & & \\
\hline
\end{tabular}

